\title{
Balkanologie
}

Balkanologie Revue d'études pluridisciplinaires

Vol. VI, $n^{\circ}$ 1-2 | 2002

Volume VI Numéro 1-2

\section{L'Ile aux Serpents : source de contentieux entre la Roumanie et l'Ukraine}

The Island of Snakes: Source of disputes between Romania and Ukraine

\section{François Després}

\section{(2) OpenEdition}

12 Journals

Édition électronique

URL : http://journals.openedition.org/balkanologie/441

DOI : 10.4000/balkanologie.441

ISSN : 1965-0582

Éditeur

Association française d'études sur les Balkans (Afebalk)

Édition imprimée

Date de publication : 1 décembre 2002

Pagination : 55-60

ISSN : 1279-7952

\section{Référence électronique}

François Després, «L'lle aux Serpents : source de contentieux entre la Roumanie et l'Ukraine », Balkanologie [En ligne], Vol. VI, n 1-2 | 2002, mis en ligne le 03 février 2009, consulté le 17 décembre 2020. URL : http://journals.openedition.org/balkanologie/441 ; DOI : https://doi.org/10.4000/ balkanologie. 441

(c) Tous droits réservés 


\section{L'ILE AUX SERPENTS : SOURCE DE CONTENTIEUX ENTRE LA ROUMANIE ET L'UKRAINE}

François Després*

Le litige entre la Roumanie et l'Ukraine à propos de l'Ile aux Serpents est aujourd'hui largement méconnu du grand public international. Pourtant, loin d'être anecdotique, ce différend revêt un caractère stratégique, étant donné sa position géographique et les ressources naturelles qui s'y trouvent. C'est pourquoi ces deux Etats y attachent encore actuellement une importance considérable, qui peut notamment apparaître disproportionnée par rapport à sa superficie. Cette île se situe en Mer Noire, au large des Bouches du Danube, plus précisément du Bras de Sulina, à environ 45 kilomètres de la ville roumaine de Sulina. Sa superficie totale est de 17 hectares.

\section{RAPPEL HISTORIQUE}

Connue dans l'Antiquité sous le nom d'Ile Blanche (ou Brillante), elle est supposée avoir accueilli la dépouille du héros de la Guerre de Troie, Achille. L'appellation actuelle est apparue au Moyen Age, sans que la présence de serpents y ait jamais été attestée. Bien que se trouvant sous la domination de l'Etat médiéval de Dobroudja puis des Principautés de Valachie et de Moldavie, elle resta complètement inoccupée. Elle fut placée sous souveraineté ottomane à partir de la fin du XVe siècle, lorsque l'Empire ottoman fit de la Mer Noire un "lac turc". Lîle avait fait l'objet d'escales de différentes flottes croisant en Mer Noire,

\footnotetext{
- Docteur en géographie (option géopolitique) de l'Université Paris VIII, diplômé de l'Institut d'Etudes Politiques d'Aix-en-Provence. Sujet de thèse : L'espace roumain, limites et affrontements. Le cas moldave. Soutenue en mai 2000.
} 
L'lle aux Serpents

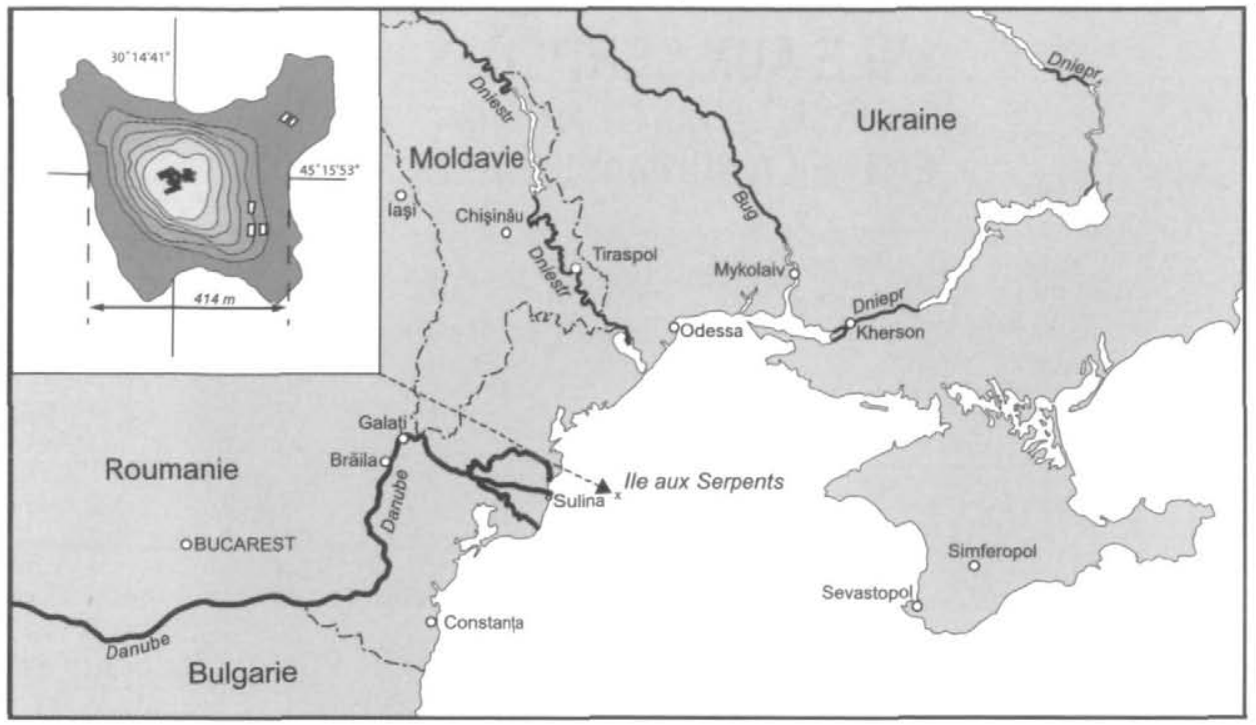

mais aucune puissance régionale n'avait jugé bon d'y installer une présence permanente. A la fin du XVIII ${ }^{\mathrm{e}}$ siècle, l'Empire russe avait le désir de s'étendre vers le Sud, c'est-à-dire dans la direction de Constantinople et des Détroits. Le contrôle de la Mer Noire constituait donc pour lui un objectif stratégique majeur.

C'est pourquoi les Russes manifestèrent ouvertement leur intérêt pour l'Ile aux Serpents lors de la conclusion à Bucarest du traité russo-ottoman de 1812. Ils considérèrent désormais l'île comme n'appartenant à aucun Etat ; aucune fortification ne pouvait y être construite. En 1817 et avec l'accord des Ottomans, les Russes déclarèrent qu'elle était désormais sous leur souveraineté. Ceci fut confirmé officiellement par le traité russo-ottoman signé à Andrinople en 1829. Le traité de Paris de 1856 la rétrocéda aux Ottomans. Le traité de San Stefano, signé le 3 mars 1878 , leur imposa de la céder à nouveau à la Russie. Celle-ci précisa qu'elle se réservait la possibilité d'un échange avec le Sud de la Bessarabie, qui se trouvait sous souveraineté roumaine. Au congrès de Berlin (13 juin-13 juillet 1878), le gouvernement roumain présenta un mémoire dans lequel il était demandé que "la Roumanie reprenne possession des îles du Danube et des Bouches du Danube, y compris l'Ile aux Serpents ". Le traité conclu à l'issue de ce congrès attribua celle-ci à la Roumanie, qui n'y installa aucune garnison. Au moment de l'application du Pacte RibbentropMolotov, l'Union Soviétique ne l'annexa pas en juin 1940. Mais sa marine y débarqua symboliquement à la fin du mois d'août 1944, après le changement d'alliance de la Roumanie. Le traité de Paris de 1947 confirma néanmoins l'appartenance de l'île à la Roumanie. Par le protocole du 4 février 1948 et sans aucune négociation préalable, l'Union Soviétique imposa à la Roumanie une mo- 
dification de leur frontière dans la zone de la Mer Noire, en l'obligeant notamment à lui céder l'Ile aux Serpents en échange d'une protection de la frontière maritime roumaine. La cession intervint officiellement par la conclusion du procès-verbal du 28 mai 1948. Il faut remarquer que les Parlements des deux Etats n'examinèrent, ni ne ratifièrent jamais ce procès-verbal. L'Union Soviétique y implanta de puissantes installations radar, à la fois de détection et de guidage de missiles. Un port pouvant accueillir des navires de moyen tonnage et des sous-marins, ainsi qu'un héliport, y furent aménagés. Les autorités soviétiques firent donc de cette île l'un des éléments clé de leur stratégie de contrôle de la Mer Noire et du détroit du Bosphore. Elles pouvaient de cette manière surveiller l'ensemble des vols de l'OTAN, ainsi que la totalité du trafic maritime. C'est pourquoi elle acquit une importance vitale pour l'Etat qui pouvait la détenir avec l'ensemble de ses installations militaires.

\section{RENAISSANCE D'UN LITIGE AU DÉBUT DES ANNÉES 1990}

Lors de la décomposition soviétique, le nouvel Etat ukrainien affirma aussitôt sa souveraineté sur l'île, y maintenant une garnison de 70 hommes et préservant les capacités opérationnelles de ses deux installations radar. Dans le nouveau contexte régional, les autorités roumaines réclamèrent à plusieurs reprises au début des années 1990 la restitution de l'lle aux Serpents. Vu l'importance stratégique de l'île et le danger qu'elle représentait potentiellement à leurs yeux, elles insistaient pour recouvrer leur souveraineté sur l'île. Des auteurs roumains estimaient en outre qu'elle était une continuité du plateau continental de la Dobroudja et était par conséquent, du point de vue géographique et géologique, indubitablement roumaine. L'Ile aux Serpents devint donc un litige territorial entre l'Ukraine et la Roumanie et une source potentielle de conflits. Lors des négociations diplomatiques entamées en 1995 en vue d'aboutir à la signature d'un traité bilatéral, la question de l'Ile aux Serpents fit notamment échouer les discussion du mois de décembre. Les propos du ministre roumain des Affaires étrangères, Teodor Meleșcanu, sur le possible recours à la Cour de Justice internationale pour régler le différend roumano-ukrainien à propos de l'île déclenchèrent immédiatement des réactions courroucées à Kiev. Le ministre ukrainien des Affaires étrangères, Ghenadi Udovenko, se déclara " surpris par la suggestion de son homologue roumain de s'adresser à la Cour de Justice internationale " et affirma : "Cela signifie que la Roumanie a des revendications territoriales sur l'Ukraine. Je peux dire de manière responsable que l'Ukraine ne fera aucune concession concernant son intégrité territoriale ". L'Ukraine rappela aussitôt en consultation son ambassadeur en poste à Bucarest. Cette manière de procéder dans le monde diplomatique équivalait 
à un coup de semonce avant la rupture totale des négociations. Lors d'une rencontre informelle à Londres quelques jours plus tard, les deux ministres eurent une " explication nécessaire " à ce sujet. Pour la partie roumaine,

la réaction du chef de la diplomatie de Kiev de rappeler son ambassadeur de Bucarest n'a été fondée que sur les informations diffusées par la presse, informations qui dénaturaient les affirmations du ministre roumain... Pour éclaircir la situation, le sténogramme de la séance parlementaire au cours de laquelle monsieur Meleșcanu a fait la déclaration (...) sera envoyé à Kiev.

La Roumanie désirait donc calmer la situation, afin que les négociations bilatérales n'échouent pas. Mais Teodor Meleşcanu déclara à nouveau au début de l'année 1996 : " Les prétentions ukrainiennes à conserver l'lle aux Serpents ne sont pas fondées. Si l'on n'arrive pas à résoudre cette question par la négociation, nous nous adresserons à la Cour de Justice internationale ". Ces nouvelles déclarations suscitèrent immédiatement de vives réactions en Ukraine. Le ministre de la Défense annonça qu'il renforcerait la présence militaire sur l'île et le maire d'Odessa, Edouard Gurvitz, après y avoir effectué une visite spectaculaire, déclara qu'il n'était " pas question d'abandonner un millimètre de son territoire aux Roumains ". Les Ukrainiens considéraient en effet que l'île faisait partie intégrante, de fait et de droit, de leur territoire. Mais au même moment et à propos des informations diffusées dans la presse roumaine concernant une activité intense observée sur l'île, l'ambassadeur ukrainien à Bucarest fit remarquer :

Ce sont pour de bon des rumeurs et elles découlent du domaine du fantastique. Sur l'île est placée une unité de gardes-frontières. "Auparavant", leur approvisionnement était assuré par la flotte maritime soviétique de la Mer Noire. Ces dernières années, personne n'a rempli ces fonctions. Pour cela, aujourd'hui, l'Ukraine a êtẻ obligée d'effectuer des opérations de reconstruction. Celles-ci sont dues à l'usure de la falaise et des différents moyens techniques; mais les travaux qui se déroulent maintenant sur l'île sont de pures opérations de réparation, d'entretien... Pour l'Ukraine, l'lle aux Serpents a une importance nationale du point de vue économique. Les années précédentes, de nombreux navires de braconnage, non seulement roumains, mais aussi de pays tiers, ont été retenus par nos gardes-frontières. Les réserves de pétrole et de gaz naturel se trouvent principalement sur le plateau continental.

Outre le sentiment national sous-tendu par un intérêt stratégique, la véritable source du litige roumano-ukrainien était donc constituée par cette présence avérée d'hydrocarbures. Il ne faut pas oublier que les deux Etats, et particulièrement l'Ukraine, cherchent à renforcer leur indépendance énergétique à l'égard de la Russie. Les négociations bilatérales se poursuivaient néanmoins dans ce contexte relativement tendu. Le nouvel ambassadeur d'Ukraine à Bucarest se montra au début du mois de février 1996 très ouvert à l'égard de la position roumaine, puisqu'il affirma que " la Roumanie et l'Ukraine ne sont pas en guerre et que le problème de l'lle aux Serpents étant de nature juri- 
dique, il pourrait être réglé si les deux parties, d'un commun accord, s'adressaient au tribunal international de La Haye ". Il soulignait, en revanche, ses craintes vis-à-vis de prétentions territoriales émanant de certains partis politiques roumains, qui exigeaient " la liquidation des conséquences du Pacte Ribbentrop-Molotov ". Les deux gouvernements faisaient face en effet, de part et d'autre, à des discours peu modérés, qu'ils devaient absolument contenir pour aboutir à la conclusion d'un traité. Les deux Etats restaient très attachés au principe de l'intangibilité des frontières existantes, ce qui ne pouvait que faciliter les pourparlers entre diplomates. La principale difficulté à propos de l'île demeurait toutefois d'ordre juridique, étant donné que les règles du droit maritime ne pouvaient s'appliquer strictement entre les deux Etats. La distance qui sépare l'île de la côte roumaine est trop courte (18 kilomètres) pour que s'applique la convention internationale de Montego Bay.

L'alternance politique qui intervint en Roumanie en décembre 1996 donna une nouvelle impulsion au processus de négociations roumano-ukrainien. L'Tle aux Serpents demeurait toutefois un objet de contentieux difficile à régler. C'est pourquoi lors d'une réunion de la fin du mois de février 1997 destinée à finaliser le projet de traité, le principal négociateur roumain, Dumitru Ceaușu, affirma :

Il est sûr que dans les questions controversées, il a fallu trouver des solutions de compromis. D'un côté et de l'autre, il y a eu des situations où il a fallu que nous renoncions à certaines positions initiales. Le traité sera accompagné d'un autre document, qui sera signé dans le même temps, document dans lequel nous établirons une procédure de résolution du problème du plateau continental de la Mer Noire. De cette manière, nous établirons une solution paquet qui créera un équilibre des engagements.

Les positions des deux Etats divergeaient encore totalement à propos de l'île. L'Ukraine considérait en effet que le problème des eaux territoriales entourant l'Ile aux Serpents devait rester en suspens et qu'il serait seulement tranché après la conclusion du traité. A l'opposé, la Roumanie proposait de négocier encore pendant deux ans; si à l'issue de cette période, les deux parties n'aboutissaient à aucun compromis, le différend devait être soumis à la Cour de Justice internationale de La Haye. Les diplomates roumains voulaient absolument convaincre l'opinion publique internationale que la délimitation du plateau continental autour de l'île devrait être effectuée selon leurs propositions. Dumitru Ceaușu considérait en mars 1997 que lîle devait revenir à la Roumanie :

Le principe agréé par la communauté internationale - concernant le tracé des frontières maritimes - est celui de la ligne médiane, l'équidistance par rapport aux points les plus avancés des rives. L'équidistance consacre le principe d'égalité entre Etats. L'lle aux Serpents se trouve dans la ligne droite de la rive roumaine et affecterait le calcul de la ligne médiane. Selon les normes internationales reconnues par toutes les instances, les formations géologiques, qui ne se prêtent pas à la vie économique propre et ne sont pas propices à l'habitat humain, n'ont pas le droit au plateau continental. L'lle aux Serpents devrait être ignorée. 


\section{VERS UNE RÉSOLUTION DU CONFLIT ?}

Mais les efforts intenses des deux diplomaties et la pression de certains Etats occidentaux (notamment ceux appartenant à l'OTAN) aboutirent à la signature du traité roumano-ukrainien de bon voisinage et de coopération le 2 juin 1997 à Constanța (le principal port roumain sur la Mer Noire). C'était un évènement sans précédent dans la région puisqu'il s'agissait du premier traité bilatéral conclu par la Roumanie avec l'un de ses Etats voisins situés à l'Est. Dans le deuxième alinéa de l'article 2 du traité susmentionné, les deux parties avaient convenu de régler de manière spécifique le litige de l'Ile aux Serpents :

Les parties contractantes... résoudront le problème de la délimitation de leur plateau continental et de leurs Zones Economiques Exclusives en Mer Noire, sur la base des principes et des procédures convenus par un échange de lettres entre les ministres des affaires étrangères, effectué dès la signature du présent traité. Les accords convenus dans cet échange de lettres entreront en vigueur simultanément avec l'entrée en application de ce traité.

En souhaitant résoudre l'ensemble de leurs litiges politiques et territoriaux, la Roumanie et l'Ukraine avaient donc décidé de faire tomber la tension qui persistait à propos de l'Ile aux Serpents. C'est notamment en partageant les gains économiques prévisibles que les deux gouvernements avaient trouvé la voie du compromis.

A l'heure actuelle, plus de cinq ans après la signature du traité roumanoukrainien, les négociations concernant l'Ile aux Serpents et la délimitation des espaces maritimes n'ont guère progressé. Il faut noter que celles-ci n'ont pas été facilitées par la découverte avérée de gisements d'hydrocarbures en juillet 2001 par la société mixte Crimean Petroleum Compagnie (créée par la firme britannique JKX OIL GAS PLC et la société ukrainienne Cernomorneftegaz), exploitables commercialement à 4000 mètre de profondeur à environ 40 kilomètres au sud de l'Ile aux Serpents. Les experts roumains ont estimé que cette zone de forage se trouvait à environ 12 kilomètres à l'est de leurs eaux territoriales, au nord de la zone contiguë. Cette possibilité de forage viole l'un des principes qui régissent les négociations entre les deux Etats, puisque ceux-ci doivent s'abstenir de toute exploitation des ressources minérales jusqu'à la fin des pourparlers. Les diplomates des deux Etats continuent à se réunir régulièrement, sans réussir toutefois à atteindre un compromis acceptable de part et d'autre. Le principal enjeu est désormais constitué par le contrôle des ressources naturelles, sources de richesses pour l'Etat exploitant. 